\title{
A Template-based UHE Neutrino Search Strategy for the Askaryan Radio Array (ARA)
}

\section{Myoungchul Kim* on behalf of the ARA Collaboration}

(a complete list of authors can be found at the end of the proceedings)

Chiba University

E-mail: mkim@icecube.wisc.edu

The Askaryan Radio Array (ARA) is a gigaton-size neutrino radio telescope located near the geographic South Pole. ARA has five independent stations designed to detect Askaryan emission coming from the interactions between ultra-high energy neutrinos ( $>10 \mathrm{PeV}$ ) and Antarctic ice. Each station includes of 16 antenna deployed in a matrix shape at up to $200 \mathrm{~m}$ deep in the ice. A simulated neutrino template, including the detector response model, was implemented in a new search technique for reducing background noise and improving the vertex reconstruction resolution. The template is used to scan through the data using the matched filter method, inspired by LIGO, looking for a low SNR neutrino signature and ultimately aiming to lower the detector's energy threshold at the analysis level. I will present the estimated sensitivity improvements to ARA analyses through the application of the template technique with results from simulation.

$37^{\text {th }}$ International Cosmic Ray Conference (ICRC 2021)

July 12th-23rd, 2021

Online - Berlin, Germany

${ }^{*}$ Presenter 


\section{Introduction}

Ultra-High Energy (UHE) neutrinos are essential particles for understanding powerful accelerators in the universe. The nature of the neutrino as a weakly interacting particle allows it to travel through space will carrying information about its origin. The detection of UHE neutrinos requires the ability to detect $\sim 1$ event per gigaton per year due to the low cross section for their interaction [1]. Thus, the radio transparent ice in Antarctica, with attenuation length of $\sim 1 \mathrm{~km}$ for radio wave, provides an optimal environment for constructing a large detector [2].

The Askaryan radio array (ARA) is a largest neutrino telescope constructed in the glacier ice near the South Pole. It is designed to detect UHE neutrinos above $10^{16} \mathrm{eV}$ by utilizing the Askaryan effect [3]. The five autonomous stations have been deployed up to $200 \mathrm{~m}$ below the Antarctic surface with a $2 \mathrm{~km}$ distance between the stations. Each station contains 20 radio frequency (RF) antennas in the 6 strings, and electronics with the data acquisition (DAQ) system at the surface. The 16 antennas, 4 antennas per string, have been deployed matrix shape for observing neutrino-induced radio signals with timing information for vertex reconstruction. The remaining 4 antennas are transmitting known pulser at a $1 \mathrm{~Hz}$ rate from $\sim 40 \mathrm{~m}$ horizontally away from the station for calibration [4]. The RF antennas are divided into two types, vertically-polarized (VPol) and horizontally-polarized (HPol), to measure the polarization of the electric field (E-field) for neutrino direction reconstruction, as shown in Fig. 1.

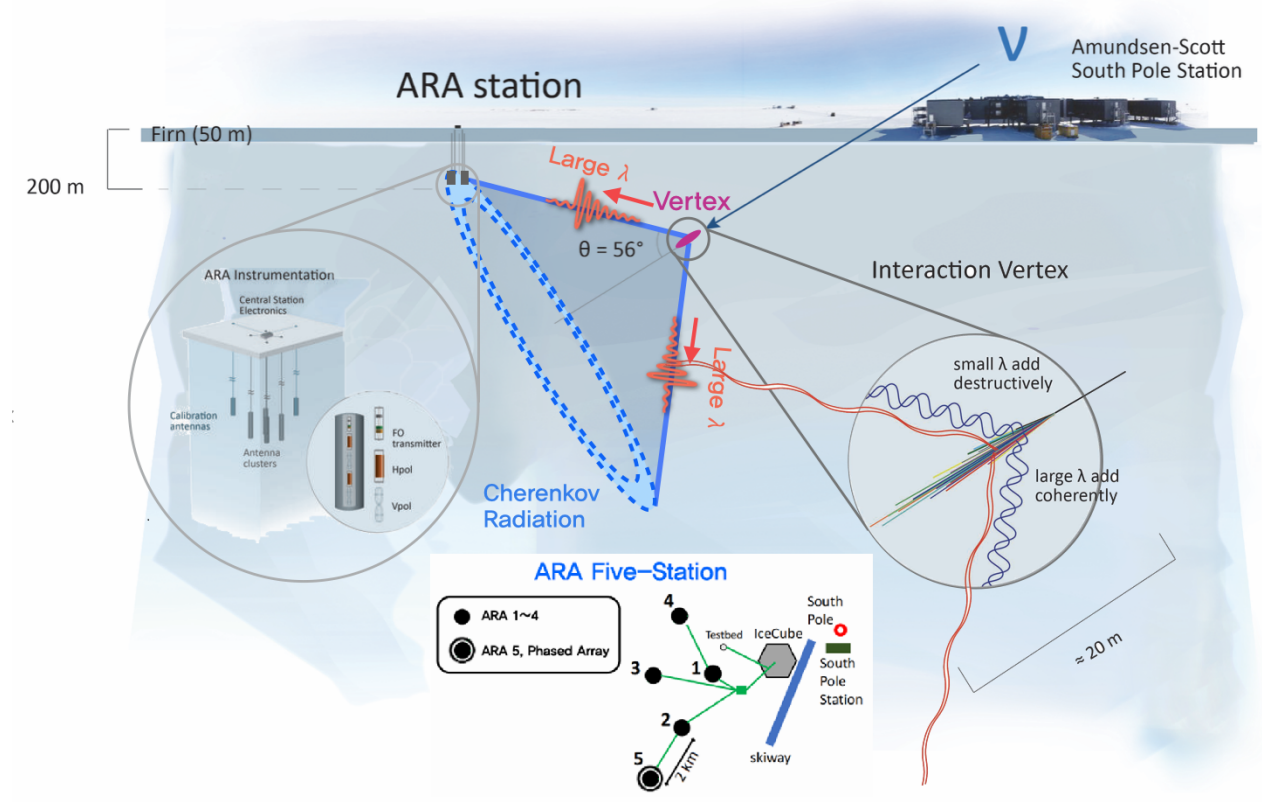

Figure 1: The schematic of the ARA detector design. A coherent radio wave is generated when a neutrino interacts with the ice. Each ARA antenna records the radio signal and is amplified in the electronics.

\section{Lowering Energy Threshold}

The amplitude of the neutrino-induced waveform is related to neutrino energy and the distance between detector and interaction position. Based on two conditions, the signal amplitude can be 
similar to the thermal noise background and anthropogenic noise from the South pole station, which can be also detected by the ARA detector. The matched filter method using a neutrino template, inspired by LIGO, is designed to distinguish low signal-to-noise ratio (SNR) signals from the noise waveforms [5]. Lowering the amplitude of neutrino signals that we can detect would increase our sensitivity.

\subsection{Matched Filter Method}

The matched filter method is used to distinguish the desired signal from the stochastic noise dominant waveforms by using the signal as a template. It is convenient for searching neutrinoinduced radio waves that have a characteristic short pulse from the dominant thermal noise background.

The data waveform is cross-correlated with the expected neutrino waveform. In the crosscorrelation, each frequency bin is weighted by the power spectral density (PSD) of the thermal noise that contains no signal spectrum. The cross-correlated results $C(t)$ can be expressed like,

$$
C(t)=4 \int_{0}^{\infty} \frac{D(f) T^{*}(f)}{P_{n}(f)} d f
$$

where $D(f)$ is a spectrum of the data waveform. In this expression, $T^{*}(f)$ is a spectrum of the time-reversed template, and $P_{n}(f)$ is PSD of thermal noise without signal. This convolution gives the location of the hidden signal as the lag between data and template.

The suppression of noise from the cross-correlation result including normalization can be expressed by

$$
\rho(t)=\frac{|C(t)|}{\sigma}
$$

where $\rho(t)$ is the weighted correlation value, which is a measure of the similarity between the hidden signal and the template in each lag time. And

$$
\sigma^{2}=4 \int_{0}^{\infty} \frac{|T(f)|^{2}}{P_{n}(f)} d f
$$

is normalizing the power of template $T^{*}(f)$ and the power of noise $P_{n}(f)$ [5][6].

\subsection{ARA Application}

The event samples need for the matched filter method come from two sources, triggered data from the detector and simulations. The RF trigger is designed to record data if it is over a power threshold that $\sim 6$ times higher than noise RMS for the analysis. The threshold is estimated by detector calibration and applied to the simulation. The detector station also records the software triggers at $1 \mathrm{~Hz}$ to sample the noise environment. The software-triggered data can be used for PSD of noise.

The template is generated by AraSim which models the antenna angular-dependent gain patterns, the system electronics, neutrino information, and the Askaryan effect [2]. There are a number of templates that depend on different parameters. The neutrino energy and the distance between the detector and the vertex can be simplified by a scale factor. But the antenna gain patterns as 
a function of zenith angle, the fraction of the energy in the electromagnetic (EM) and hadronic (HAD) showers, and different Askaryan effects need to be included as template parameters. In this proceeding, one template generated with a zenith angle for the incoming neutrino of $90^{\circ}$, as EM shower only, and Askaryan signal at $55.8^{\circ}$ from the shower axis was used for analyzing simulated data [7]. The analysis will be continued in the future with multiple template parameters.

\section{Simulation}

\subsection{Noise Tuning}

The agreement between data and simulation is validated by the software-triggered data. Since the matched filter method takes into account the spectral shape of the data waveforms and the template, simulating an accurate detector response is crucial. In order to estimate thermal noise behavior including the detector response, the $10 \%$ of the noise data was used. The distribution of spectral amplitudes in a given bin can be described by a Rayleigh of form

$$
f(x ; \alpha)_{f r e q}=\frac{x}{\alpha^{2}} e^{-x^{2} /\left(2 \alpha^{2}\right)}
$$

where $x$ is amplitude and $\alpha$ is mode. To verify the simulated thermal noise behavior is matches the data, the distributions of simulated noise with different fit parameters were compared with a sample of noise from the data. The Fig. 2 shows the agreement between the spectral amplitudes of noise data and simulated noise based on the fit results at a specific frequency [8].

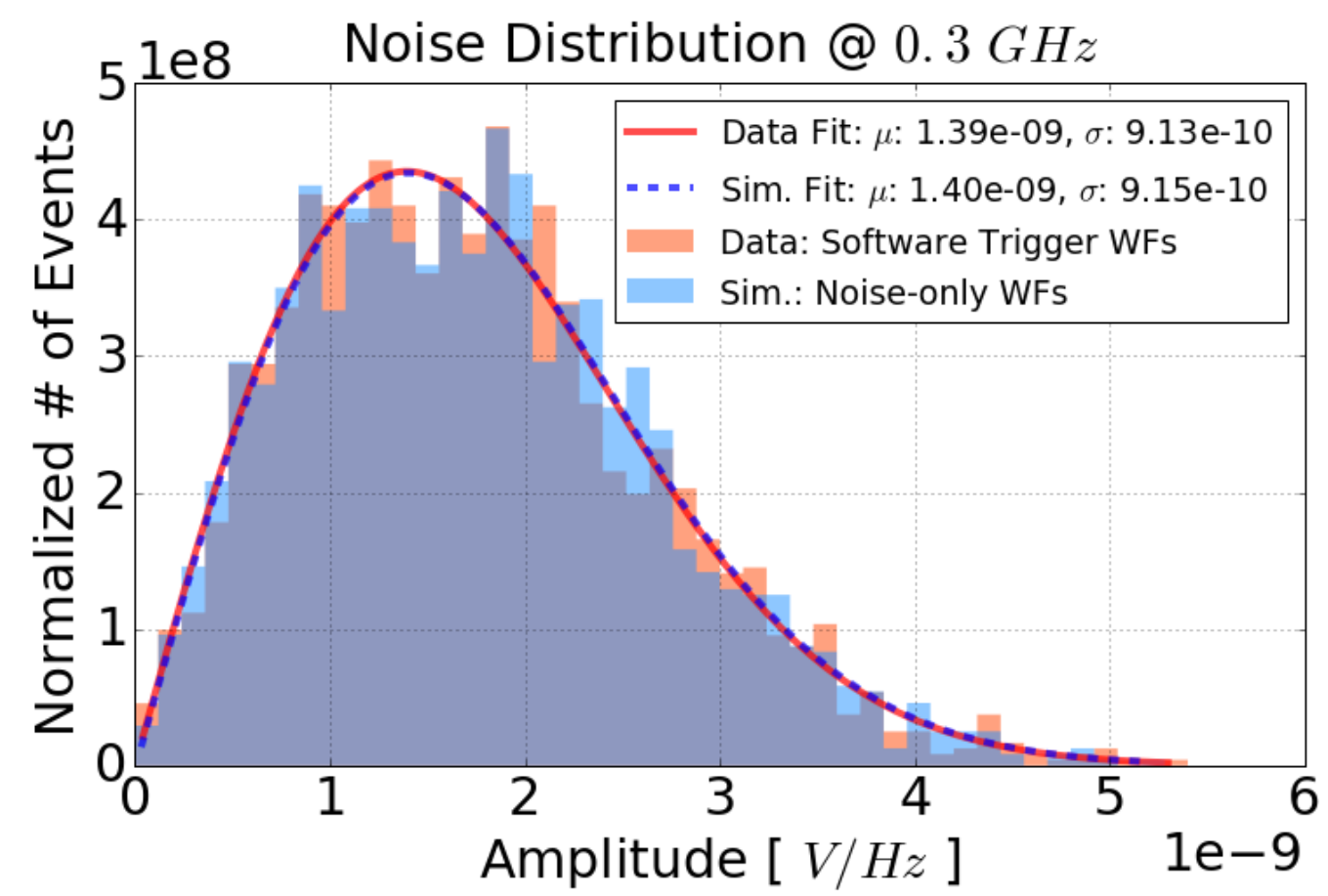

Figure 2: Agreement between data and simulation spectrum. Data in the distribution is only recorded by software triggers. Simulated noise is generated using results from fitting to the data. 
In this proceeding, the fit results are possibly including anthropogenic noise from the South pole activity. That would be removed in the future for studying the behavior of noise on a sample that is more clean. The power threshold value implemented in the simulation also needs to be optimized based on the agreement between simulation and data.

\subsection{Neutrino Template}

The neutrino template in this proceeding is generated by AraSim. The model for neutrino emission is based on [7]. The detector response is simulated using an empirical model based on measurements of the antennas and electronics. A zenith angle of 90 degrees, with maximal gain at all frequency range, is selected for this template. The electronic system is obtained by deconvolving the estimated in-ice noise from the in-situ noise spectrum calculated from a Rayleigh fit [4]. Since each antenna channel has a unique shape to its electronics system shape in frequency-domain, the different templates are prepared for each channel. The Fig. 3 shows two examples of the template waveforms of two polarizations based on Channels 0 and 8 on the A2 station.

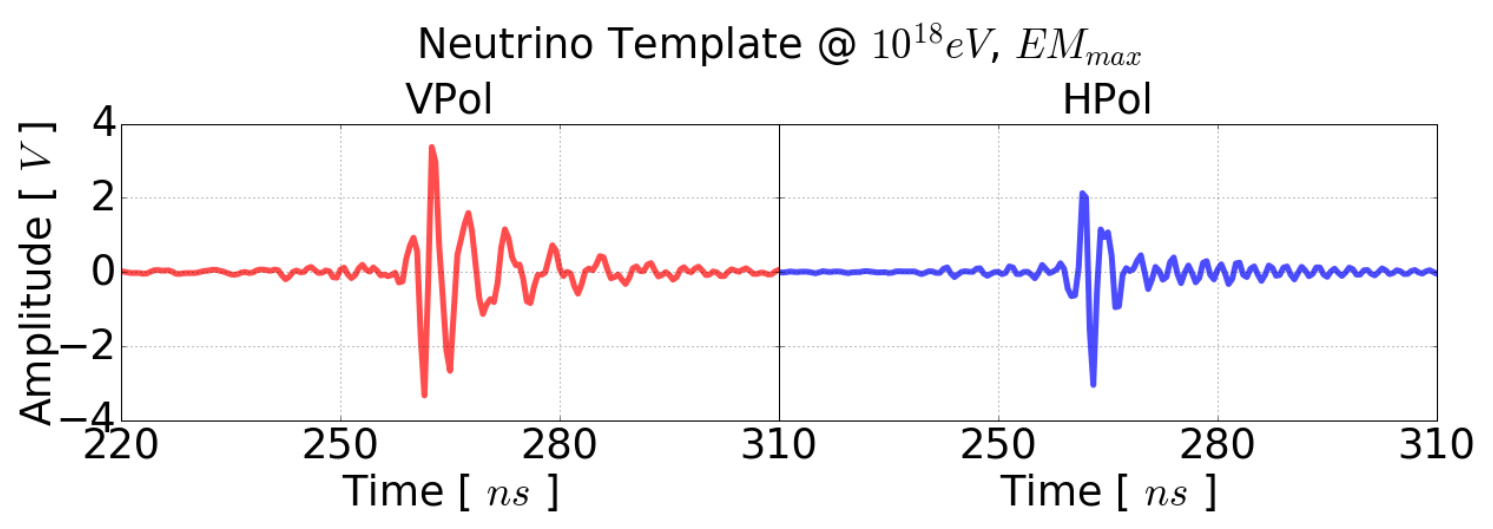

Figure 3: The simulated template waveform. Both waveforms are including the electronics calculated by noise tuning and empirical antenna gain. The left waveform is from the VPol, and the right is from the HPol antenna.

\section{Analysis}

The matched filter method is applied to individual waveforms generated from the simulation. Two sets of simulations were produced. The noise-only simulation is for estimating the background level and the PSD of noise used as weights. The simulation that sum signal with noise is taken as the signal sample on which the matched filter method can be tested. The PSD of noise is calculated by fitting the simulated noise distribution in each frequency. The Fig. 4 is an example of the result of the matched filter method searching. The template is correlated with data in the frequency domain and normalized. So, it is only taking into account the shape of the template spectrum. In this proceeding, only the maximum weighted correlation value $\rho(t)_{\max }$ was chosen for scoring the individual waveform. If a double-pulse was presented in the waveform, the only highest peak is chosen. 


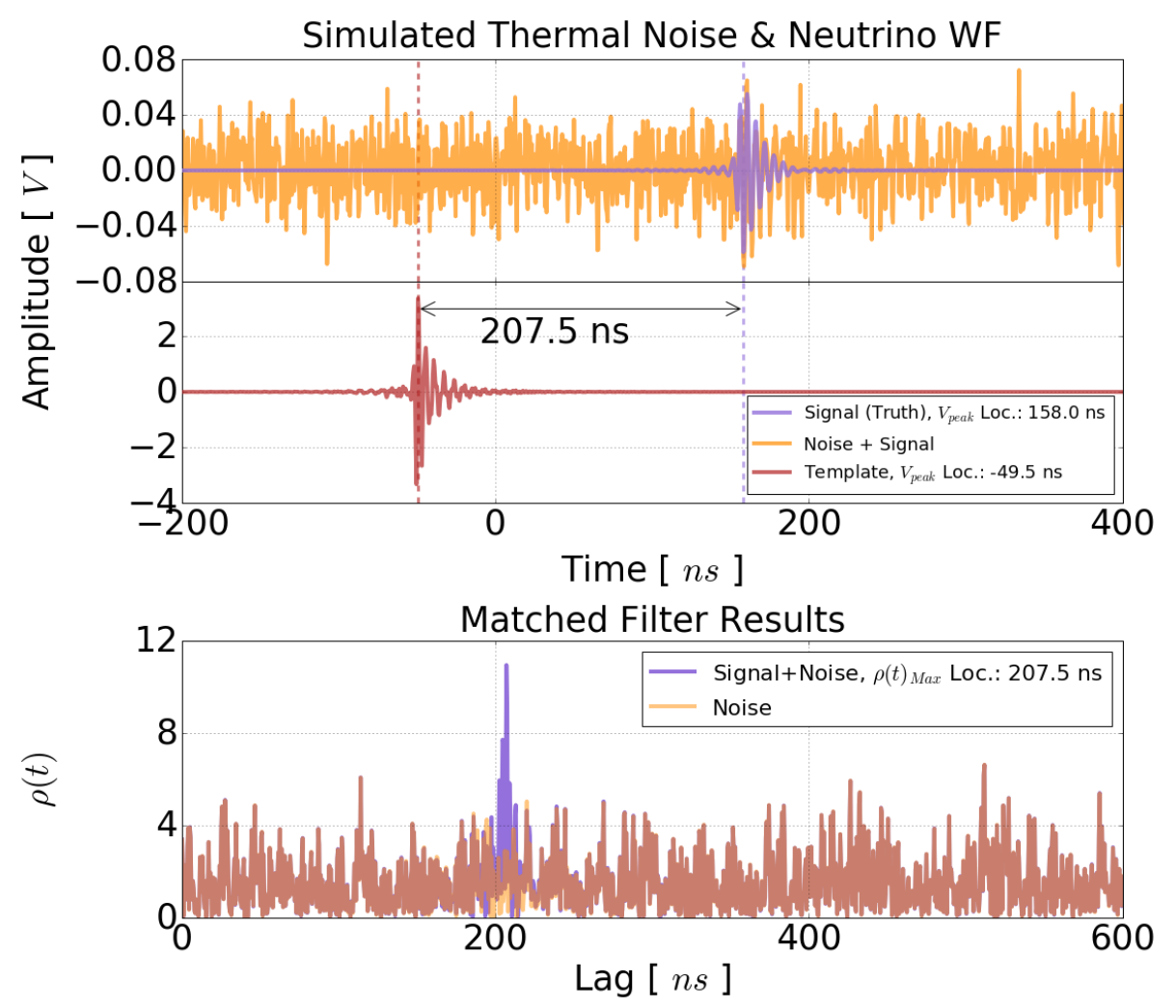

Figure 4: The results of a low-SNR signal search with matched filter method. Top: location of the hidden signal in the noise and template. The hidden signal is generated $\sim 4 \mathrm{~km}$ far from the station and having a property of $10^{20} \mathrm{eV}$ and viewing angle of $61.8^{\circ}$ from the neutrino direction. Bottom: The results of crosscorrelation with the waveform including hidden signal and without it. The $\rho(t)_{\max }$ of the hidden signal is $\sim 2$ times higher than what is typically obtained with noise, and the corresponding lag value is consistent with the time difference between the signals in the template and the simulated waveform.

\subsection{Analysis Cut}

The analysis cut is set by the estimated three-station livetime based on six years of the A2 and A3 stations and 2 years livetime from the A5 station. Half a million noise events were generated and tested using the matched filter method and scaled to the number of events in our data-set acquired at a $5 \mathrm{~Hz}$ rate. The neutrino simulation is weighted by the Kotera max neutrino flux model, and applied to the matched filter method [9]. The data from each antenna channel produces $\rho(t)_{\max }$ by correlating with the template. A third highest correlation value, $\rho(t)_{\max , 3 r d}$, from 16 antennas is chosen to evaluate each event. Based on the exponential fit, the analysis cut was made for separating noise from signal events. The Fig. 5 shows the distribution of $\rho(t)_{\max , 3 r d}$ from two simulation sets. If the $\rho(t)_{\max , 3 r d}$ is passed the cut, then the event is a signal candidate.

In this proceeding, results from each individual antenna is taken into account for the analysis cut. In the future, the analysis cut value will be calculated at an event-wise level by adding up the results for $\rho(t)$ from the individual antennas after removing the expected arrival time delay. The summed $\rho(t)$ would be like 

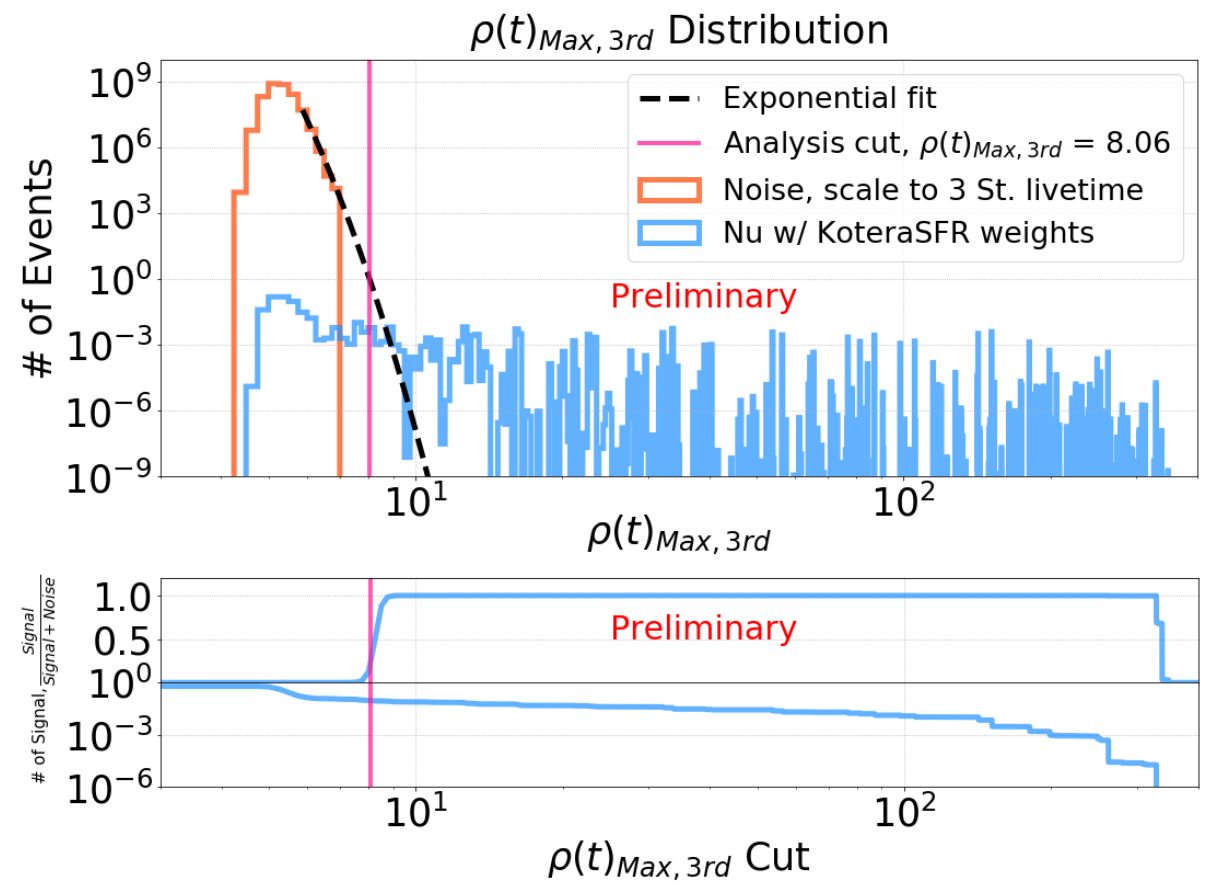

Figure 5: The distribution of $\rho(t)_{\max , 3 r d}$ results. Top: The noise is scaled up to three-station livetime, and the Kotera max flux model weights the signal. Bottom: The signal purity, a ratio of signal to signal plus noise, and number of signal by integrating over the $\rho(t)_{\max , 3 r d}$ cut.

$$
\rho_{\text {sum }}(t)=\sqrt{\frac{\left|C_{1}\left(t+\tau_{1}\right)+C_{2}\left(t+\tau_{2}\right)+\ldots+C_{16}\left(t+\tau_{16}\right)\right|^{2}}{\sigma_{1}^{2}+\sigma_{2}^{2}+\ldots+\sigma_{16}^{2}}}
$$

Where $\tau$ is the expected arrival time from the individual antennas [5]. It would take into account the total $\rho(t)$ from each antenna and amplify the trace of the neutrino signal in that event. Vertex reconstruction would be added to the cut to increase significance.

\subsection{Event Rate}

The analysis level of the event rate is calculated by the cut parameters. Both the trigger and the analysis level are based on the Kotera max neutrino flux model. The Fig. 6 shows the event rate based on different energies and the ratio of the analysis level to the trigger level. Based on the ratio plot, vertex reconstruction is required as another analysis cut to contain the low-SNR signal identified by the matched filter method. Furthermore, the rates will improve when we add a more sophisticated filtering cut, accurate livetime and the quality cut on the DAQ level is implemented.

\section{Summary and Outlook}

The matched filter method gives a strategy to search for low-SNR signal in a radio detector. The actual data will be tested against the simulated neutrino template. So, the understanding of the detector responses in the noise background will be crucial. In order to test actual data against a template, the analysis cut must be improved. The event-wise correlation value, $\rho_{\text {sum }}(t)_{\text {max }}$, obtained 


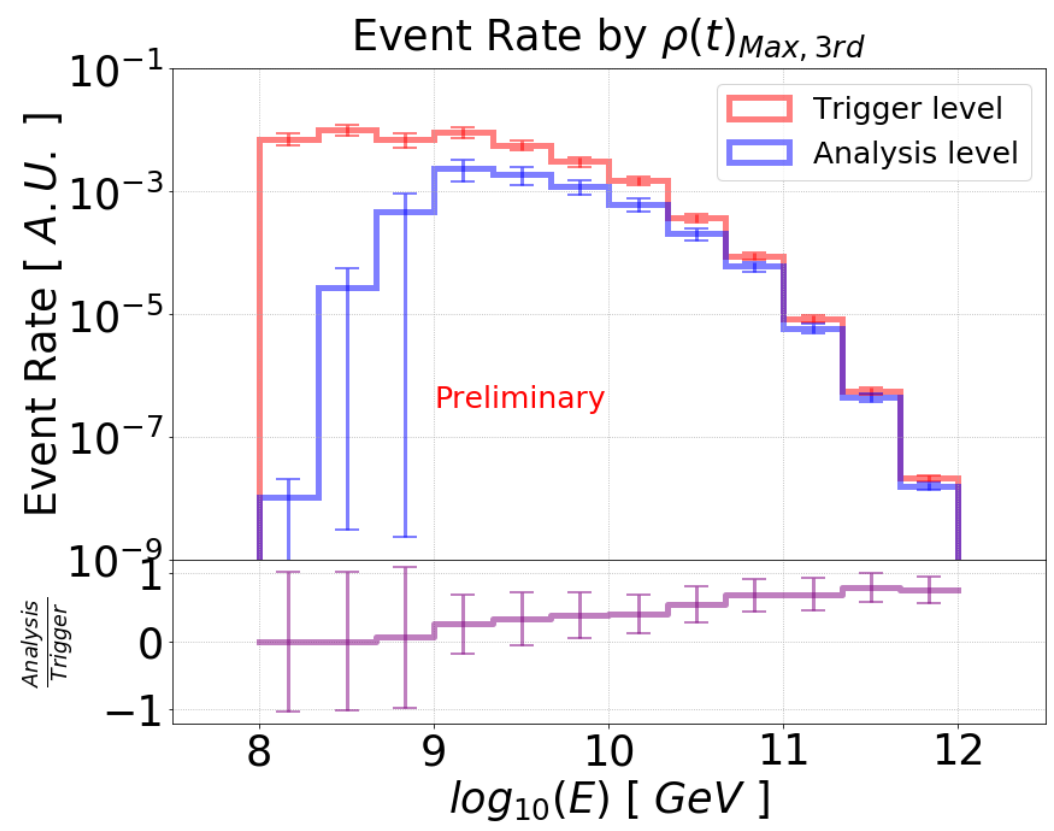

Figure 6: The event rate and the ratio of the analysis level to the trigger level with different energy spectrum. The analysis level is calculated by the events that are passed the cut.

by increasing the number of templates including double-pulse signals and vertex reconstruction will be implemented in the future for improving the cut and sensitivity.

\section{References}

[1] R. Gandhi, C. Quigg, M. H. Reno, and I. Sarcevic, Astroparticle Physics 5, 81 (1996).

[2] P. Allison et al. (ARA), Astropart. Phys. 35457 (2012).

[3] G. A. Askaryan, JETP 14, 441 (1962).

[4] P. Allison et al. (ARA), Phys. Rev. D 93082003 (2016).

[5] B.Abbott et al. (LIGO Scientific Collaboration), Phys. Rev. D 69122001 (2004).

[6] L. A. Wainstein and V. D. Zubakov, Extraction of signals from noise (Prentice-Hall, Englewood Cliffs, NJ, 1962).

[7] J. Alvarez-Muiz and E. Zas, Physics Letters B 411, 218 (1997).

[8] P. Allison et al. (ARA), astro-ph. HE e-print: arXiv:1404.5285v2.

[9] A. V. Olinto, K. Kotera, and D. Allard, Nucl. Phys. B, Proc. Suppl. 217, 231 (2011). 


\section{Full Author List: ARA Collaboration}

P. Allison ${ }^{15}$, S. Archambault ${ }^{17}$, J.J. Beatty ${ }^{15}$, M. Beheler-Amass ${ }^{4}$, D.Z. Besson ${ }^{10,13}$, M. Beydler ${ }^{4}$, C.H. Chen ${ }^{2}$, P. Chen ${ }^{2}$, Y.C. Chen ${ }^{2}$, B.A. Clark ${ }^{16}$, W. Clay ${ }^{8}$, A. Connolly ${ }^{15}$, L. Cremonesi ${ }^{6}$, P. Dasgupta ${ }^{18}$, J. Davies ${ }^{6}$, S. De Kockere ${ }^{9}$, K.D. de Vries ${ }^{9}$, C. Deaconu ${ }^{8}$, M. A. DuVernois ${ }^{4}$, J. Flaherty ${ }^{15}$, E. Friedman ${ }^{3}$, R. Gaior ${ }^{17}$, J. C. Hanson ${ }^{19}$, K. Hanson ${ }^{4}$, N. Harty ${ }^{1}$, B. Hendricks ${ }^{11,12}$, K.D. Hoffman ${ }^{3}$, B. Hokanson-Fasig ${ }^{4}$, E. Hong ${ }^{15}$, S.Y. Hsu ${ }^{2}$, J.J. Huang ${ }^{2}$, M.-H. Huang ${ }^{2}$, K. Hughes ${ }^{8}$, A. Ishihara ${ }^{17}$, A. Karle ${ }^{4}$, J.L. Kelley ${ }^{4}$, R. Khandelwal ${ }^{4}$, K.-C. Kim $^{3}$, M.-C. Kim ${ }^{17}$, R. Krebs ${ }^{11,12}$, I. Kravchenko ${ }^{14}$, Y. Ku ${ }^{11,12}$, C.Y. Kuo ${ }^{2}$, K. Kurusu ${ }^{17}$, U.A. Latif ${ }^{10,9}$, A. Laundrie ${ }^{4}$, H. Landsman ${ }^{7}$, M.-Y. Lu ${ }^{4}$, T.-C. Liu ${ }^{2}$, B. Madison ${ }^{10}$, K. Mase ${ }^{17}$, T. Meures ${ }^{4}$, J. Nam ${ }^{2}$, A. Novikov ${ }^{10}$, R.J. Nichol ${ }^{6}$, G. $\mathrm{Nir}^{7}$, A. Nozdrina ${ }^{10}$, E. Oberla ${ }^{8}$, A. OMurchadha ${ }^{4}$, J. Osborn ${ }^{14}$, Y. Pan ${ }^{1}$, C. Pfendner ${ }^{5}$, N. Punsuebsay ${ }^{1}$, J. Roth ${ }^{1}$, P. Sandstrom ${ }^{4}$, D. Seckel ${ }^{1}$, Y.-S. Shiao ${ }^{2}$, A. Shultz ${ }^{10}$, D. Smith ${ }^{8}$, J. Torres ${ }^{15}$, S. Toscano ${ }^{18}$, J. Touart ${ }^{3}$, N. van Eijndhoven ${ }^{9}$, G.S. Varner ${ }^{22}$, A. Vieregg ${ }^{8}$, M.-Z. Wang ${ }^{2}$, S.-H. Wang ${ }^{2}$, Y.H. Wang ${ }^{2}$, S.A. Wissel ${ }^{11,12,20,21}$, C. Xie ${ }^{6}$, R. Young ${ }^{10}$, S. Yoshida 17 ,

${ }^{1}$ Dept. of Physics, University of Delaware, Newark, DE 19716

${ }^{2}$ Dept. of Physics, Grad. Inst. of Astrophys., Leung Center for Cosmology and Particle Astrophysics, National Taiwan University, Taipei, Taiwan

${ }^{3}$ Dept. of Physics, University of Maryland, College Park, MD 20742

${ }^{4}$ Dept. of Physics, University of Wisconsin-Madison, Madison, WI 53706

${ }^{5}$ Dept. of Physics and Astronomy, Denison University, Granville, Ohio 43023

${ }^{6}$ Dept. of Physics and Astronomy, University College London, London, United Kingdom

${ }^{7}$ Weizmann Institute of Science, Rehovot, Israel

${ }^{8}$ Dept. of Physics, Enrico Fermi Institue, Kavli Institute for Cosmological Physics, University of Chicago, Chicago, IL 60637

${ }^{9}$ Vrije Universiteit Brussel, Brussels, Belgium

${ }^{10}$ Dept. of Physics and Astronomy, University of Kansas, Lawrence, KS 66045

${ }^{11}$ Center for Multi-Messenger Astrophysics, Institute for Gravitation and the Cosmos, Pennsylvania State University, University Park, PA 16802

${ }^{12}$ Dept. of Physics, Pennsylvania State University, University Park, PA 16802

${ }^{13}$ Moscow Engineering Physics Institute, Moscow, Russia

${ }^{14}$ Dept. of Physics and Astronomy, University of Nebraska, Lincoln, Nebraska 68588

${ }^{15}$ Dept. of Physics, Center for Cosmology and AstroParticle Physics, The Ohio State University, Columbus, OH 43210

${ }^{16}$ Dept. of Physics and Astronomy, Michigan State University, East Lansing, Michigan 48824

${ }^{17}$ Dept. of Physics, Chiba University, Chiba, Japan

${ }^{18}$ Université Libre de Bruxelles, Science Faculty CP230, B-1050 Brussels, Belgium

${ }^{19}$ Dept. Physics and Astronomy, Whittier College, Whittier, CA 90602

${ }^{20}$ Dept. of Astronomy and Astrophysics, Pennsylvania State University, University Park, PA 16802

${ }^{21}$ Physics Dept., California Polytechnic State University, San Luis Obispo, CA 93407

${ }^{22}$ Dept. of Physics and Astronomy, University of Hawaii, Manoa, HI 96822

\section{Acknowledgements}

We thank the National Science Foundation Office of Polar Programs and Physics Division for their generous support through NSF OPP-902483, Grant NSF OPP-1002483, Grant NSF 1607555, Grant NSF OPP-1359535, Grant NSF OPP-1404212, and Grant NSF OPP-2013134, and Grant NSF 2019597. 